\title{
Acridones: Strongly Emissive HIGHrISC Fluorophores
}

\section{Supporting Information}

Kristoffer A. Thom, ${ }^{1}$ Felix Wieser, ${ }^{1}$ Kevin Diestelhorst, ${ }^{1}$ Anna Reiffers, ${ }^{1}$ Constantin Czekelius, ${ }^{2}$ Martin Kleinschmidt, ${ }^{3}$ Mario Bracker, ${ }^{3}$ Christel M. Marian, ${ }^{3}$ and Peter Gilch ${ }^{1 *}$

${ }^{1}$ Institut für Physikalische Chemie, Heinrich-Heine-Universität Düsseldorf, Universitätsstr. 1, 40225 Düsseldorf, Germany

${ }^{2}$ Institut für Organische Chemie und Makromolekulare Chemie, Heinrich-Heine-Universität Düsseldorf, Universitätsstr. 1, 40225 Düsseldorf, Germany

${ }^{3}$ Institut für Theoretische Chemie und Computerchemie, Heinrich-Heine-Universität Düsseldorf, Universitätsstr. 1, 40225 Düsseldorf, Germany

*E-mail: gilch@hhu.de

\section{Contents}

1. Experimental Procedures

2. Synthesis and characterization

3. Nanosecond transient absorption on NMA-dF

4. Time-resolved emission spectra of NMA-dF + DCB mixtures

5. Nanosecond transient absorption on NMA-dF + DCB mixtures

6. Steady-state fluorescence spectroscopy on NMA-dF + DCB mixtures

7. Quantum chemical computations

References 


\section{Experimental procedures}

Samples

NMA, TX (97\%) and thymidine ( $\geq 99.0 \%)$ were purchased from Sigma-Aldrich. DCB (> 99.0\%) and AC (> 98.0\%) were supplied by $\mathrm{TCl}$. Coumarin 102 and 1,4-bis(5-phenyloxazol-2-yl)-benzene (POPOP) were purchased from Radiant Dyes Laser. The solvents used for spectroscopic experiments were of HPLC gradient grade. All commercially available chemicals were used as received.

\section{Common conditions}

All measurements were carried out at room temperature $\left(\approx 20^{\circ} \mathrm{C}\right)$. Except for steady-state absorption and femtosecond transient absorption spectra all sample solutions were deoxygenized by purging them prior to and during the experiments with argon or nitrogen (Air Liquide, 99.999\%). Gases were saturated with the corresponding solvent to prevent concentration changes of the sample solutions. Unless stated otherwise fused silica cells (Hellma Analytics) with $d=1 \mathrm{~cm}$ path length were used. Sample solutions were pumped through flow cells to avoid signal contributions of photo-products.

\section{Steady-state spectroscopy}

Absorption spectra were recorded with a two-beam Lambda 19 spectrometer (Perkin Elmer). Steadystate fluorescence spectra were obtained on a FluoroMax-4 from HORIBA Scientific. All spectra were corrected for the spectral sensitivity of the instruments.

Fluorescence data on DCB were acquired using a home-build Kerr-setup ${ }^{1}$ due to its higher sensitivity in the UV region. The sample was excited at $267 \mathrm{~nm}$ and pumped through a $1 \mathrm{~mm}$ flow cell. The fluorescence light passed the Kerr gate in its open state (parallel polarizers) in $180^{\circ}$ geometry. The spectral sensitivity of the instrument was corrected for. To determine the fluorescence quantum yield of DCB, thymidine served as reference $\left(\Phi_{f l}=10^{-4}\right.$ in water $\left.{ }^{2}\right)$.

CIE coordinates were determined using a Qmini Wide VIS spectrometer (RGB Photonics) while irradiating the sample with a hand-held UV lamp at $385 \mathrm{~nm}$.

\section{Correction of steady-state fluorescence spectra}

In the steady-state sensitization experiments described in this study, absorptions were employed for which a linear relation between fluorescence and absorption breaks down. Therefore, a calibration curve was recorded with the same instrumental settings $\left(\lambda_{\text {exc }}=266 \mathrm{~nm}\right.$, spectral bandpass of $1 \mathrm{~nm}$ for both, excitation and emission) as in the actual experiments. The calibration curve was acquired by measuring various solutions with different absorptions and plotting the spectrally integrated fluorescence signals $S_{\text {int }}$ as a function of the absorption $A$. For solutions with $A / d<0.1 / \mathrm{cm}$ the signal increases almost linear with the absorption while for higher optical densities the signal grows slower showing a peak intensity for optical densities $\approx 0.9 / \mathrm{cm}$. For even higher values the fluorescence signal decreases due to the inner filter effect. This behavior was fitted with a trial function adapted from ref. ${ }^{3}$.

$$
S_{\text {int }}(A)=f \cdot\left[10^{-\frac{A}{d}\left(x_{m}-\frac{\Delta x}{2}\right)}-10^{-\frac{A}{d}\left(x_{m}+\frac{\Delta x}{2}\right)}\right]
$$


Herein, $f$ is a scaling factor and $A$ the absorption of the solution for complete light passage of a cuvette with thickness $d=1 \mathrm{~cm}$. For the right-angle geometry employed here, fluorescence emission originating from the center of the cuvette located at $x_{m}$ ought to dominate the signal. The width of the region contributing is denoted $\Delta x$. The fit yields $0.49 \mathrm{~cm}$ and $0.16 \mathrm{~cm}$ for $x_{m}$ and $\Delta x$, respectively. Fluorescence spectra obtained in the sensitization experiments were corrected by dividing them by the right-hand side of eq. S1. Hereby, the total absorption given by the sum of NMA-dF and DCB contributions was inserted as $A$.

\section{Time-resolved fluorescence spectroscopy}

Time-correlated single photon counting was performed on a FluoTime 300 (PicoQuant) with a supercontinuum picosecond laser (SuperK Extreme and SuperK Extend-UV, NKT Photonics) as excitation source running at $19.5 \mathrm{MHz}$. Emission was detected under magic angle conditions until a peak value of 10000 counts was achieved. The sample solution featured an optical density of $<0.1 / \mathrm{cm}$ at the excitation wavelength.

Time-resolved fluorescence data on longer time scales were obtained with a home-built setup. A pulsed Nd:YAG laser (Spitlight 600, Innolas) tuned to $266 \mathrm{~nm}$ served as excitation source, providing pulses of $12 \mathrm{~ns}$ FWHM at a repetition rate of $10 \mathrm{~Hz}$. At the sample location the laser light featured an energy per pulse of $125 \mu \mathrm{J}$ and a diameter of $0.5 \mathrm{~mm}$. The emitted fluorescence light was collected at $54^{\circ}$ before being dispersed in a spectrograph (SpectraPro 308, Acton Research) with a custom grating blazed at $410 \mathrm{~nm}(121.6 \mathrm{l} / \mathrm{mm})$ and detected by a gated iCCD camera (PI-MAX, Princeton Instruments). The delay between excitation and detection was controlled electronically (PTG, Princeton Instruments) and enabled delay times from the nanosecond to millisecond regime. To achieve proper signal intensities even at long delay times the integration time of the detector as well as the number of accumulations were increased for longer delay times. Furthermore, signals were amplified by raising the gain voltage of the microchannel plate. The signals of those pixel rows of the CCD chip on which the fluorescence light impinged on were added up (200 rows) and the resulting spectra were spectrally integrated over the emission band of interest. To correct for different baselines the integrated signals recorded at another 200 pixel rows of the CCD chip where no fluorescence light impinged onto were subtracted from the raw data points. Finally, the pre-corrected data points were divided by the number of accumulations and by the integration time applied. Compensation for different gain voltage was accomplished by acquiring a calibration curve with same instrumental settings at different gain voltages $U_{M C P}$. An exponential dependence according to eq. S2 between the fluorescence intensity $S_{\text {int }}$ and the amplification was found.

$$
S_{\text {int }}\left(U_{M C P}\right)=a \cdot e^{b \cdot U_{M C P}}
$$

For NMA-dF in methanol $a=1041263$ and $b=0.01564$ resulted with $U_{M C P}$ ranging from 0 to 255 . Correction of this dependence was achieved by dividing each data point by the right-hand-side of eq. S2. 


\section{Nanosecond flash photolysis}

Nanosecond transient absorption data were acquired with a LKS.60 spectrometer from Applied Photophysics in a right-angle geometry. A Nd:YAG laser (Spitlight 600, Innolas) with a repetition rate of $1 \mathrm{~Hz}$ and a pulse duration of $12 \mathrm{~ns}$ (FWHM) served as the excitation source. The output wavelength was frequency-quadrupled to $266 \mathrm{~nm}$. The average pulse energy amounted to $\approx 3 \mathrm{~mJ}$. Probe light was provided by a flashed xenon lamp (L2273, Hamamatsu). After passing the flow cell ( $2 \mathrm{~mm}$ path length in pump and $10 \mathrm{~mm}$ path length in probe direction), the light was dispersed in a grating monochromator and detected by a photomultiplier (1P28, Hamamatsu). To obtain transient spectra, time traces were collected from 250 to $620 \mathrm{~nm}$ in $5 \mathrm{~nm}$ steps and averaged 10 times. For sensitization experiments time traces at selected wavelengths were averaged 100 times.

\section{Femtosecond transient absorption}

The setup is described in detail elsewhere. ${ }^{4}$ Pump pulses were tuned to $400 \mathrm{~nm}$ by second harmonic generation of the fundamental of a Ti:Sa amplifier system featuring a repetition rate of $1 \mathrm{kHz}$ and a pulse width of $150 \mathrm{fs}$. The energy of a pump pulse amounted to $1.2 \mu \mathrm{J}$. Transient absorption was probed by a supercontinuum generated in $\mathrm{CaF}_{2}$. Relative polarization of pump and probe beams was set to magic angle conditions. At the sample location their respective diameters amounted to $280 \mu \mathrm{m}$ and $30 \mu \mathrm{m}$. Solutions were pumped through a $0.5 \mathrm{~mm}$ flow cell having an optical density at the excitation wavelength of $1.75 / \mathrm{mm}$. For every position of the delay line ranging from -1 ps to about $3.4 \mathrm{~ns} 2000$ spectra were accumulated and averaged over four scans. Raw data were corrected for the chirp and solvent contributions.

\section{Data analysis}

For the determination of 0-0 energies and Stokes shifts fluorescence spectra were converted from constant wavelength bandpass to constant wavenumber bandpass by multiplying with $\lambda^{2}{ }^{5}$ Absorption and fluorescence spectra were further corrected for their intrinsic frequency dependence by multiplying by $\tilde{v}^{-1}$ and $\tilde{v}^{-3}$, respectively. ${ }^{6}$ 0-0 energies were then obtained as the intersection of the corrected absorption and fluorescence spectra normalized to their maxima. Strickler-Berg analysis was performed according to ref. ${ }^{7-8}$, in which only the bands lowest in energy entered the analysis. 


\section{Synthesis and characterization}

Reagents and solvents were purchased from Acros, Sigma-Aldrich, Fisher Scientific, or Fluorochem. Chemicals were used without further purification or purified according to standard laboratory methods. Solvents were dried using the solvent purification system MP-SPS 800 from M.Braun.

Reactions were monitored by thin-layer chromatography (TLC) using Macherey-Nagel silica gel plates ALUGRAM ${ }^{\circledast}$ Xtra SIL G/UV 254 (0.20 mm thickness) and visualized by UV light. Chromatographic purification of products was performed on Macherey-Nagel $60 \mathrm{M}(0.04-0.063 \mathrm{~mm})$ silica gel.

${ }^{1} \mathrm{H}-,{ }^{13} \mathrm{C}-$, and ${ }^{19} \mathrm{~F}-\mathrm{NMR}$ spectra were recorded on Bruker Avance III 300 and 600 NMR spectrometer. Chemical shifts are reported in parts per million (ppm) with respect to TMS using the residual solvent signal as reference. The order of citation in parentheses is a) multiplicity ( $s=\operatorname{singlet}, d=$ doublet, $\mathrm{m}=$ multiplet), b) coupling constants, c) number of protons, and d) assignment. Coupling constants $(J)$ were reported in Hertz $(\mathrm{Hz})$. If not described differently the NMR-spectra were measured at $298 \mathrm{~K}$.

IR spectra were recorded using a Jasco FT/IR-6200 spectrometer. Samples were measured as $\mathrm{KBr}$ pellets. The absorption bands were given in wavenumbers $\left(\mathrm{cm}^{-1}\right)$. High-resolution mass spectra (HRMS) were measured with a Bruker Daltonics UHR-QTOF maXis 4G. Melting points were recorded on a Büchi B-540.

2,7-Difluoroacridin-9(10H)-one (AC-dF) was prepared by Ullmann-type coupling from 4-fluoroaniline and 2-bromo-5-fluoro-benzoic acid, followed by intramolecular Friedel-Crafts acylation according to literature precedence. ${ }^{9-10}$

\section{2,7-Difluoro-10-methylacridin-9(10H)-one (NMA-dF)}<smiles>Cn1c2ccc(F)cc2c(=O)c2cc(F)ccc21</smiles>

$\mathrm{N}$-Alkylation of the acridone was done following a modified protocol by Inagaki and coworkers. ${ }^{11}$

2,7-Difluoroacridin-9(10H)-one (AC-dF, $260 \mathrm{mg}, 1.12 \mathrm{mM}$ ) was suspended in dry DMF (10 mL) and sodium hydride (60\% in mineral oil, $113 \mathrm{mg}, 2.83 \mathrm{mM}$ ) added in portions at RT. Methyl iodide (175 $\mu \mathrm{L}, 399 \mathrm{mg}, 2.81 \mathrm{mM}$ ) was added to the bright yellow suspension and stirring at RT continued for 6 h. Saturated $\mathrm{NaHCO}_{3}$-Solution $(50 \mathrm{~mL})$ was carefully added to the green solution and the mixture extracted with dichloromethane $(3 \times 50 \mathrm{~mL})$. The combined organic layers were washed with water $(50 \mathrm{~mL})$ and brine $(50 \mathrm{~mL})$. After drying over sodium sulfate, all volatiles were removed in vacuo. Flash column chromatography (silica gel, hexane/ethyl acetate 1:1 to 1:2) gave the product as a canary yellow solid (235 mg, $0.958 \mathrm{mmol}, 86 \%$ ). 
Mp $=245{ }^{\circ} \mathrm{C} .{ }^{1} \mathrm{H}-\mathrm{NMR}\left(600 \mathrm{MHz}, \mathrm{DMSO}-\mathrm{d}_{6}\right): \delta[\mathrm{ppm}]=7.88(\mathrm{~m}, 4 \mathrm{H}, \mathrm{C}(1 / 8)-H$ and $\mathrm{C}(3 / 6)-H) ; 7.70(\mathrm{~m}$, $2 \mathrm{H}, \mathrm{C}(4 / 5)-H) ; 3.91$ (s, $3 \mathrm{H}, \mathrm{Me}) .{ }^{13} \mathrm{C}-\mathrm{NMR}\left(151 \mathrm{MHz}, \mathrm{DMSO}-\mathrm{d}_{6}\right): \delta[\mathrm{ppm}]=175.1,156.9(\mathrm{~d}, J=241.0 \mathrm{~Hz})$; 138.9; 122.5 (d, $J=24.8 \mathrm{~Hz}) ; 121.4(\mathrm{~d}, J=6.1 \mathrm{~Hz}) ; 119.2(\mathrm{~d}, J=7.7 \mathrm{~Hz}) ; 110.1$ (d, $J=22.0 \mathrm{~Hz}) ; 34.4$. ${ }^{19} \mathrm{~F}-\mathrm{NMR}\left(564 \mathrm{MHz}, \mathrm{DMSO}-\mathrm{d}_{6}\right): \delta[\mathrm{ppm}]=-$ 121.3. IR (film), $\widetilde{v}\left[\mathrm{~cm}^{-1}\right]: 3061,2923,1606,1584,1503,1277$, 1176, 1163, 926, 808, 779. HRMS (ESI): $\mathrm{m} / z$ calculated for $\mathrm{C}_{14} \mathrm{H}_{10} \mathrm{~F}_{2} \mathrm{NO}[\mathrm{M}+\mathrm{H}]^{+}:$246.0725; found: 246.0730 .

NMR Spectra

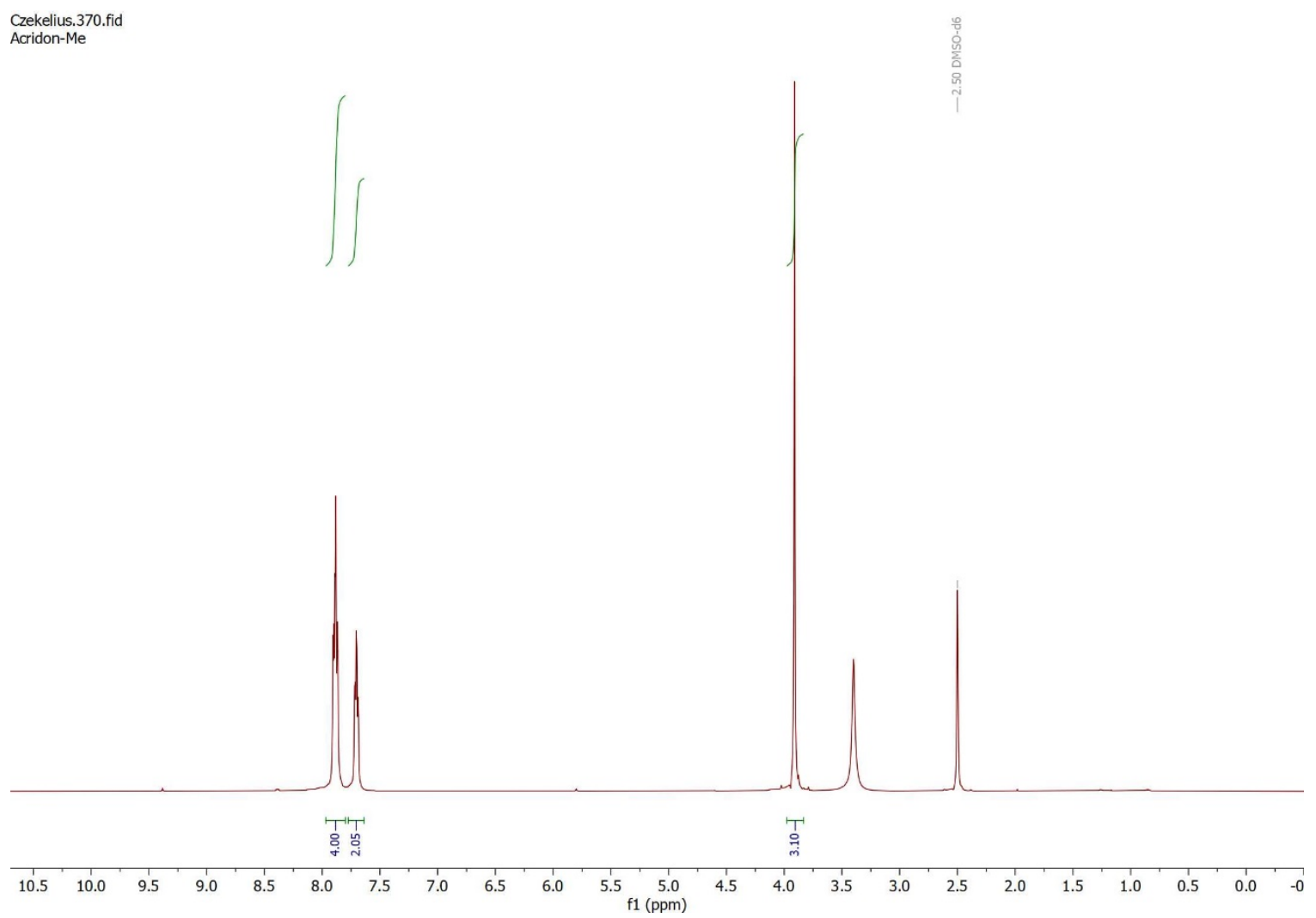

Figure S1: ${ }^{1} \mathrm{H}$ NMR spectrum (298K, $\left.600 \mathrm{MHz}, \mathrm{DMSO}-\mathrm{d}_{6}\right)$ of 2,7-difluoro-10-methylacridin-9(10H)-one (NMA-dF). 


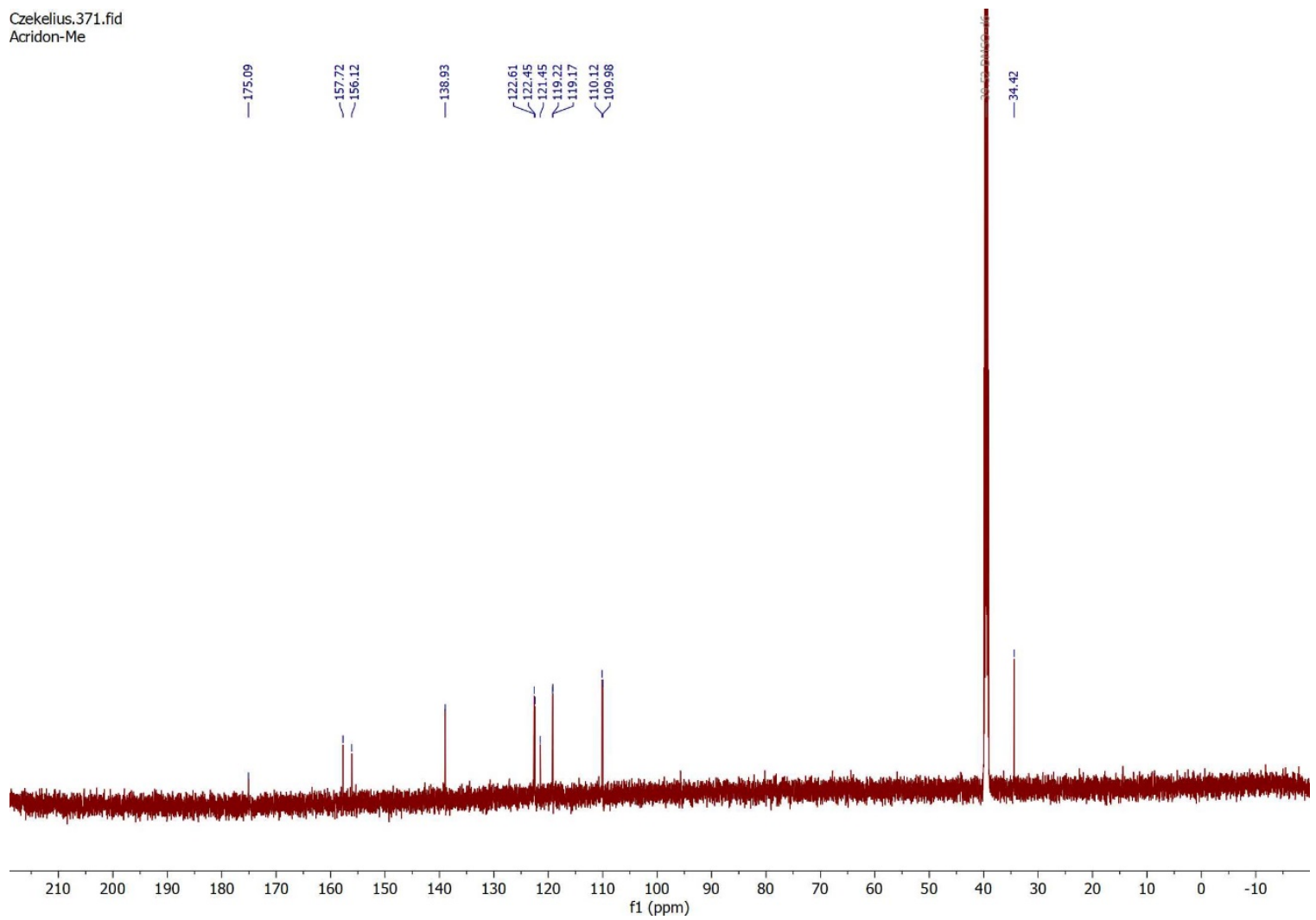

Figure S2: ${ }^{13} \mathrm{C}$ NMR spectrum (298K, $151 \mathrm{MHz}$, DMSO-d $\mathrm{d}_{6}$ ) of 2,7-difluoro-10-methylacridin-9(10H)-one (NMA-dF).
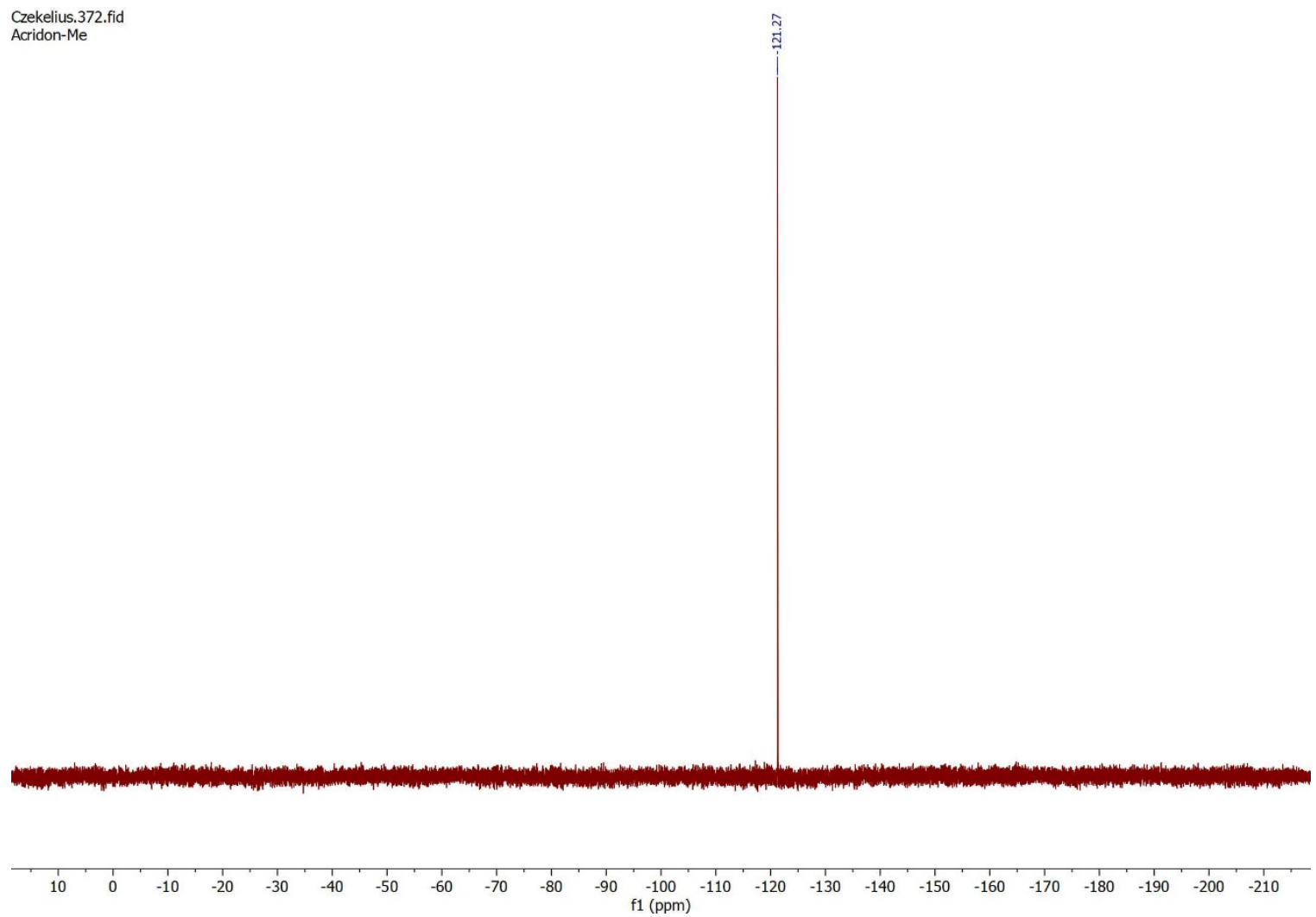

Figure S3: ${ }^{19} \mathrm{~F}$ NMR spectrum (298K, $564 \mathrm{MHz}$, DMSO-d $_{6}$ of 2,7-difluoro-10-methylacridin-9(10H)-one (NMA-dF). 


\section{Nanosecond transient absorption on NMA-dF}

The triplet quantum yield of NMA-dF in methanol was retrieved from nanosecond transient absorption spectra as described in ref. ${ }^{8}$. The spectrum (Figure $\mathrm{S} 4$ ) features two transient absorption bands peaking at $575 \mathrm{~nm}$ and around $280 \mathrm{~nm}$. Between $375 \mathrm{~nm}$ and $430 \mathrm{~nm}$ a ground state bleach is discernable. To determine the triplet quantum yield, the stationary ground state spectrum was scaled into the GSB of the transient spectrum to receive a scaling factor, which relates the molar absorption coefficient to the amplitude of the transient spectra. A difference absorption coefficient of the triplet absorption at $575 \mathrm{~nm}$ of around $20000 \mathrm{M}^{-1} \mathrm{~cm}^{-1}$ was estimated.

Based on the modified Lambert-Beer law

$$
\Delta A=\Phi_{T} \cdot \Delta \varepsilon \cdot c^{*} d
$$

the triplet yield $\Phi_{T}$ was calculated from the measured signal amplitude $\Delta A$ and the difference absorption coefficient $\Delta \varepsilon$. The latter one was derived from the amplitude of the ground state bleach. $c^{*}$ represents the concentration of initially excited molecules and $d$ the (effective) pathlength. The product $c^{*} d$ was determined by measuring thioxanthone $\left(\Phi_{T}=0.6, \Delta \varepsilon=26000 \mathrm{M}^{-1} \mathrm{~cm}^{-1}\right)^{12-13}$ in methanol as reference under the same conditions. For NMA-dF a triplet yield of $\Phi_{T}^{N M A}=0.11( \pm 0.03)$ as the average of two independent runs resulted.

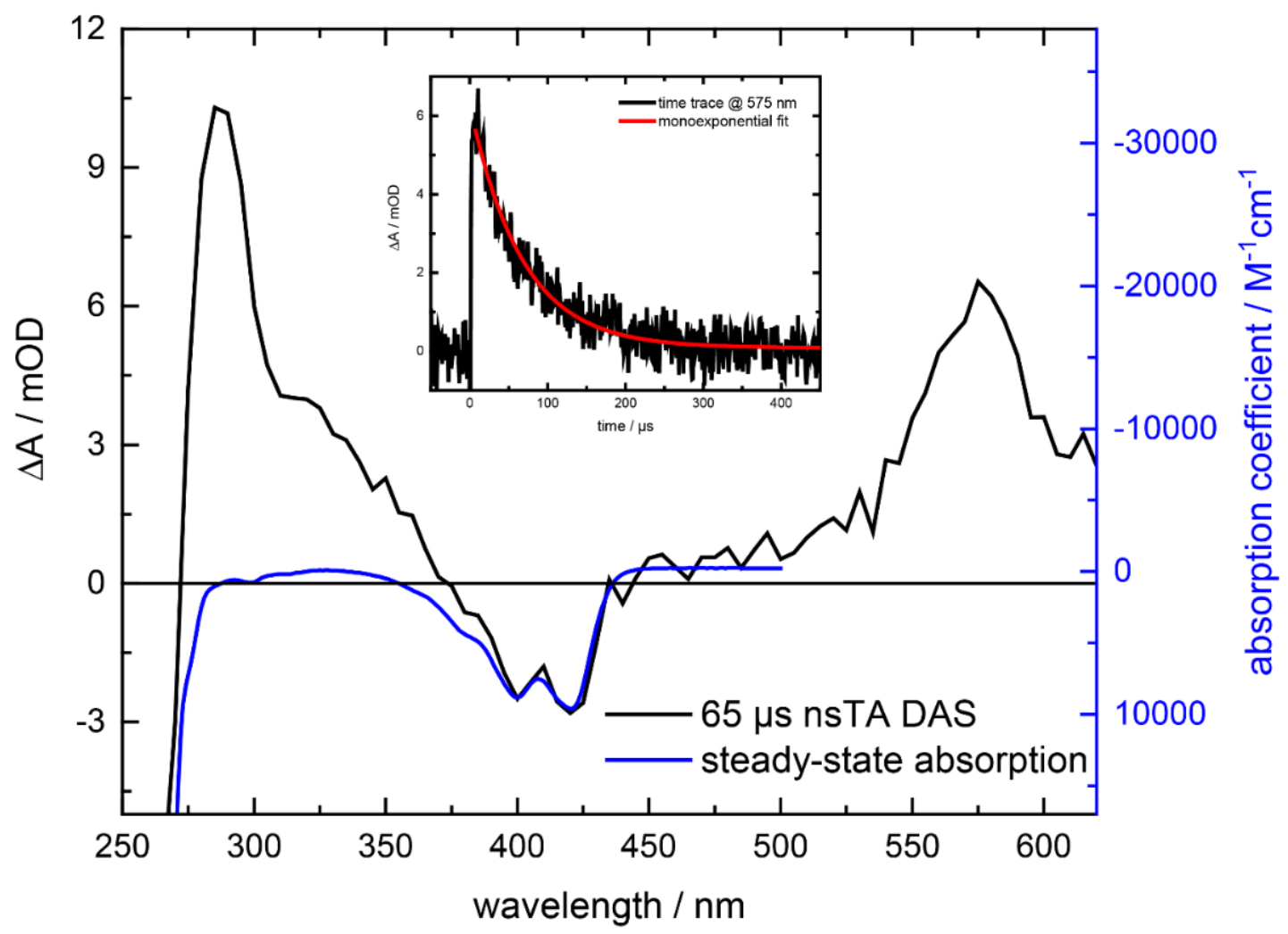

Figure S4: Nanosecond transient absorption spectrum of NMA-dF in methanol (0.02 mM, black). The steadystate absorption (blue) was inverted, shifted, and scaled to fit into the ground state bleach. Inset: time trace at $575 \mathrm{~nm}$. 
From measurements at different concentrations of NMA-dF covering two orders of magnitude the intrinsic rate constant $k_{0}^{E m}$ and the selfquenching constant $k_{\text {self }}^{E m}$ were obtained from a linear fit of the observed triplet lifetime $\tau_{T}$ against the concentration [Emitter].

$$
\frac{1}{\tau_{T}}=k_{0}^{E m}+k_{\text {self }}^{E m} \cdot \text { [Emitter] }
$$

The $\mathrm{T}_{1}$ state of NMA-dF exhibits an intrinsic lifetime of $74 \mu \mathrm{s}\left(k_{0}=1.3 \cdot 10^{4} \mathrm{~s}^{-1}\right)$ and a selfquenching rate constant $k_{\text {self }}$ of approximately $8.1 \cdot 10^{7} \mathrm{M}^{-1} \mathrm{~s}^{-1}$.

\section{Time-resolved emission spectra of NMA-dF + DCB mixtures}

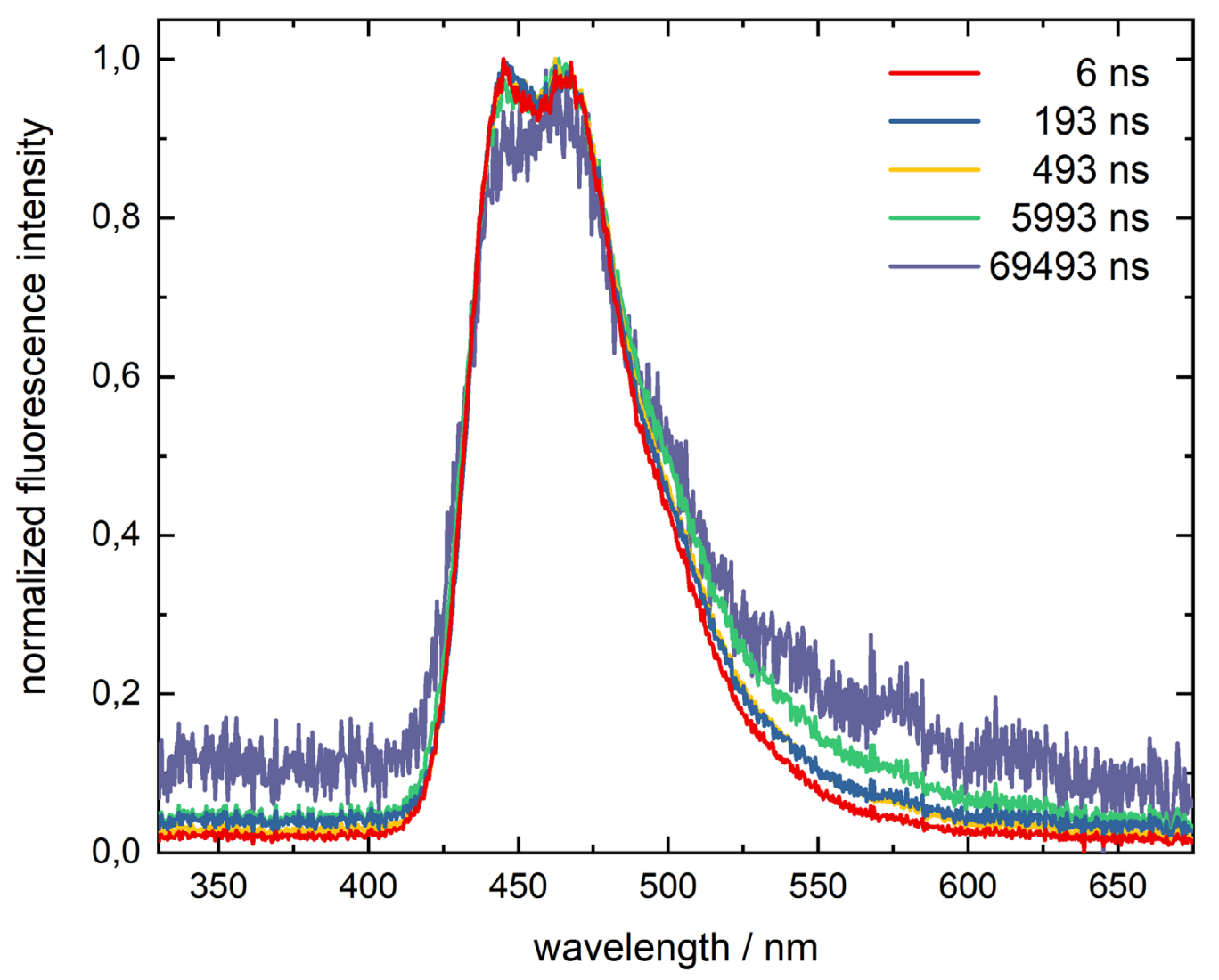

Figure S5: Emission spectra of NMA-dF in presence of DCB at various delay times as received from the timeresolved experiment. The sample solutions (nitrogen-purged methanol) were excited with $266 \mathrm{~nm}$ laser pulses. 


\section{Nanosecond transient absorption on NMA-dF + DCB mixtures}

Time traces for NMA-dF and NMA-dF + DCB solutions are compared in Figure S6. Time traces are given for a probe wavelength of $575 \mathrm{~nm}$ where the $T_{1}$ absorption of the emitter peaks (cf. Figure S4). In absence of the sensitizer, a single-exponential decay with a time constant of $34 \mu \mathrm{s}$ is observed. After adding DCB to the solution this decay remains unaffected while a delayed rise featuring a time constant of $550 \mathrm{~ns}$ becomes discernable. According to eq. 2 of the main text this corresponds to a quenching constant of $9.8 \cdot 10^{9} \mathrm{M}^{-1} \mathrm{~s}^{-1}$ in good agreement to the one obtained from the nsFl experiment.

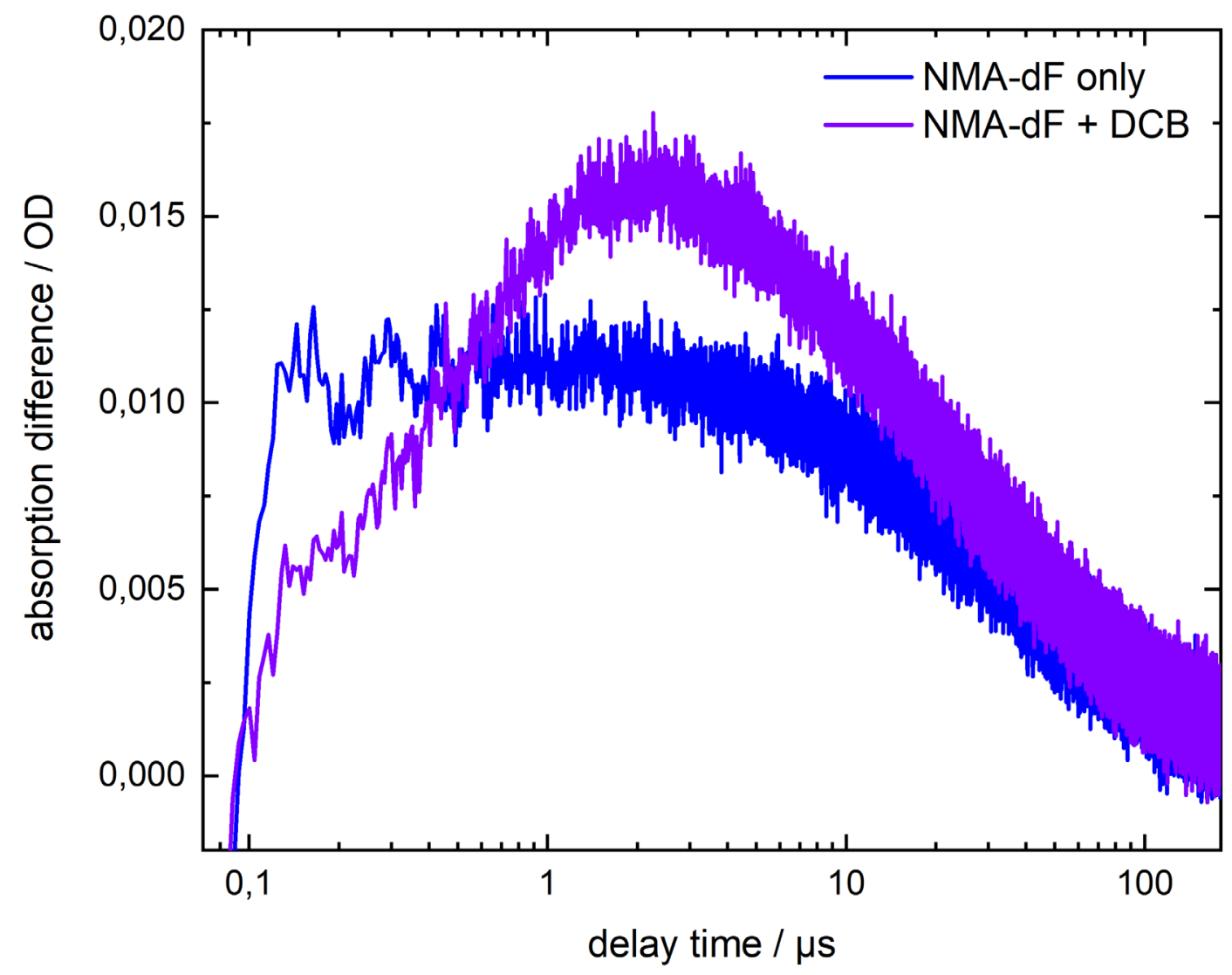

Figure S6: Nanosecond transient absorption time traces of NMA-dF $(0.14 \mathrm{mM})$ in absence (blue) and presence of DCB (purple) dissolved in nitrogen purged methanol. The sample solutions were excited with $266 \mathrm{~nm}$ laser pulses and the absorption change probed at $575 \mathrm{~nm}$. 


\section{Steady-state fluorescence experiments on NMA-dF + DCB mixtures}

In the steady-state experiments, argon-purged solutions of NMA-dF were excited at $266 \mathrm{~nm}$ (Figure S7, left). When adding DCB to the solution, the fluorescence signal of NMA-dF corrected for the absorbed light flux (cf. Experimental Procedures) decreases. This decrease is a consequence of the inner filter effect of DCB. If no energy transfer between DCB and NMA-dF occurred at all, the fractional decrease of the NMA-dF emission would be given by the part of light flux absorbed by DCB. However, the signal decrease actually observed is less pronounced than the fraction of light absorbed implies. This shows that a part of the energy absorbed by DCB is transferred to NMA-dF and causes fluorescence emission of this compound.
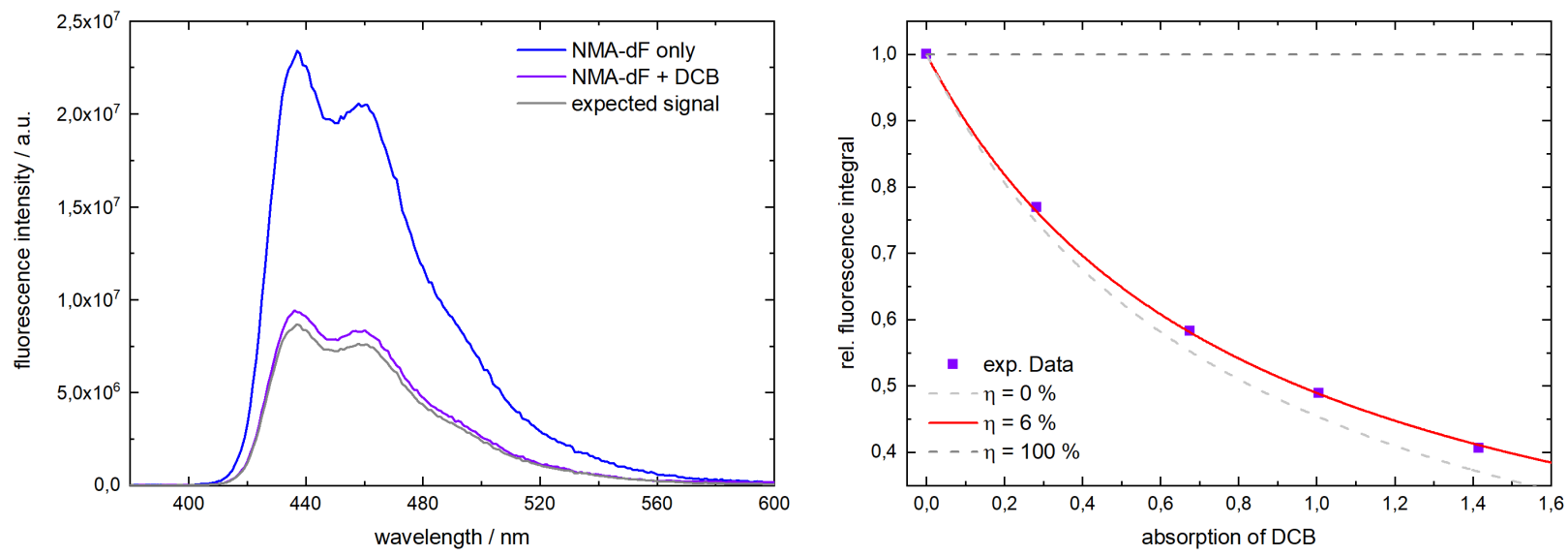

Figure S7: Steady-state sensitization experiment. Left: Steady-state fluorescence spectra of NMA-dF (0.025 mM) in absence (blue) and presence of DCB (purple) dissolved in argon purged methanol. The gray dashed spectrum represents the expected fluorescence signal based on the fraction of light absorbed by NMA-dF within the NMA$\mathrm{dF}+\mathrm{DCB}$ mixture without considering an energy transfer. Right: Spectrally integrated fluorescence of NMA-dF + DCB mixtures as a function of the absorption of DCB (purple dots) and a fit according to eq. $S 6$ for $\eta=0.06$ (red line). Simulations for $\eta=0.0$ and $\eta=1$ are shown as well (dashed lines). Spectra depicted on the left correspond to the data point for $A^{D C B}=1.42$ in the right graph.

For a quantitative assessment the following procedure was used: The fluorescence quantum yield $\varphi_{f l}^{E m+S e n s}$ of an NMA-dF + DCB mixture can be separated into a direct and a sensitized part (eq. S5). The first term contains the fraction of light absorbed by the emitter and its fluorescence quantum yield $\varphi_{f l}^{E m}$. In the second term, one finds the fraction of light absorbed by the sensitizer, the quantum yield $\varphi_{f l}^{E m}$ and the efficiency $\eta$ for an energy transfer populating the emitting state of NMA-dF.

$$
\varphi_{f l}^{E m+\text { Sens }}=\underbrace{\frac{A^{E m}}{A^{E m}+A^{S e n s}} \cdot \varphi_{f l}^{E m}}_{\text {direct }}+\underbrace{\frac{A^{\text {Sens }}}{A^{E m}+A^{\text {Sens }}} \cdot \eta \cdot \varphi_{f l}^{E m}}_{\text {sensitized }}
$$


Rearranging eq. S5 yields the expression used for the steady-state analysis of the HIGHrISC behavior. The left hand side of this expression equals the spectrally integrated fluorescence signal of the emitter in presence of the sensitizer divided by the respective emitter only integral.

$$
\frac{\int S^{\text {Emitter+Sens }}}{\int S^{\text {Emitter }}}=\frac{\varphi_{f l}^{\text {Emitter+Sens }}}{\varphi_{f l}^{\text {Emitter }}}=\frac{A^{\text {Emitter }}}{A^{\text {Emitter }}+A^{\text {Sens }}}+\frac{A^{\text {Sens }}}{A^{\text {Emitter }}+A^{\text {Sens }}} \cdot \eta
$$

The efficiency $\eta$ may be determined by conducting a set of experiments in which $A^{\text {Emitter }}$ is kept constant, whereas $A^{\text {Sens }}$ is varied by successively adding DCB to the solution (Figure S7, right). Hereby, $\eta$ was the only variable parameter. The resulting fit describes the experimental behavior well. For an NMA-dF concentration of $0.025 \mathrm{mM}$ an efficiency of $0.064( \pm 0.004)$ results. All experimental values are well above the limit for $\eta=0$, stressing that indeed an energy transfer takes place.

Note that the efficiency $\eta$ depends on the emitter concentration (see main text). The value of 0.064 given here refers to a rather low concentration. An extrapolation to high concentrations based on eq. 5 (main text) yields an efficiency $\eta_{\infty}$ of 0.18 , which matches the value determined by timeresolved spectroscopy.

\section{Quantum chemical calculations}

Geometry optimizations were conducted with the Turbomole program ${ }^{14}$ using density functional theory (DFT) with the B3LYP functional for the ground state and time-dependent DFT (TDDFT) for the excited states, additionally applying the Tamm-Dancoff approximation (TDDFT-TDA) in the triplet manifold. The TZVP basis set from the Turbomole library was used throughout.

For the numerical computation of vibrational frequencies, the SNF program was used. ${ }^{15}$ Vibrational overlaps for emission and absorption spectra, as well as for intersystem crossing (ISC) rate constants were obtained by the Vibes program in its time-dependent version. ${ }^{16}$

Vertical excitation energies at fixed geometries were computed with the DFT/MRCI program. ${ }^{17-}$

${ }^{18}$ The original Hamiltonian of Grimme and Waletzke was used with standard parameters and a selection threshold of 1.0 Hartree. The core electrons were kept frozen, the initial reference space comprised all single and double excitations of 12 electrons in 12 orbitals and was subsequently optimized to contain the most important configurations of all roots. Spin-orbit matrix elements (SOMEs) were obtained from $\mathrm{DFT} / \mathrm{MRCl}$ wavefunctions by the SPOCK program ${ }^{19}$ applying the spin-orbit operator in mean-field approximation. ${ }^{20}$ Derivatives of the SOMEs were obtained numerically and used for the calculation of the El-Sayed forbidden $\mathrm{T}_{2}-\mathrm{S}_{1}$ ISC.

Internal conversion (IC) rate constants, were obtained by a code recently developed in our group, computing non-adiabatic coupling matrix elements (NACMEs) by taking numerical derivatives of the wave function overlaps and subsequently calculating the IC rates via a modified version of the Vibes program. $^{21}$

Molecular geometries of the ground state and the two lowest excited singlet and three triplet states were calculated in vacuum. All of these correspond to true minima on the potential energy 
surface (PES). The influence of solvation in methanol was estimated by computing the ground state geometry in an implicit solvation environment (Conductor Like Screening Model (COSMO) as implemented in Turbomole) and adding two explicit methanol molecules as hydrogen-bridge donors at the carbonyl group. The vertical DFT/MRCl excitation spectrum obtained this way was compared to the corresponding spectrum in vacuum and the solvation shift of the states of interest subsequently applied to the corresponding states at the other (vacuum) geometries (Table 1). These shifts were also applied in the numerical derivation of the wavefunction overlaps for the NACMEs.

Table 1: Vertical excitation energies at different geometries. Energies for the ground state refer to the optimized vacuum geometries. Energies given for excited states are estimated based on the solvent shifts between vacuum and $\mathrm{MeOH}$. Values are given in $\mathrm{eV}$.

\begin{tabular}{|l|r|r|r|r|r|r|r|r|}
\hline & \multicolumn{1}{|c|}{ vac } & \multicolumn{2}{|c|}{$\mathrm{MeOH}$} & \multicolumn{5}{|c|}{$\mathrm{MeOH}$} \\
\hline Geometry & \multicolumn{1}{|c|}{$\mathrm{S}_{0}$} & \multicolumn{1}{|c|}{$\mathrm{S}_{0}$} & \multicolumn{1}{|c|}{ shift } & \multicolumn{1}{c|}{$\mathrm{S}_{1}$} & \multicolumn{1}{c|}{$\mathrm{S}_{2}$} & $\mathrm{~T}_{1}$ & $\mathrm{~T}_{2}$ & $\mathrm{~T}_{3}$ \\
\hline $\mathrm{S}_{0}$ & 0 & 0 & & 0.14 & 0.41 & 0.14 & 0.43 & 0.44 \\
\hline $\mathrm{S}_{1}\left({ }^{1} \pi \pi^{*}\right)$ & 3.30 & 3.17 & -0.13 & 3.06 & 3.32 & 3.07 & 3.33 & 3.36 \\
\hline $\mathrm{S}_{2}\left({ }^{1} \mathrm{n} \pi^{*}\right)$ & 3.37 & 3.90 & +0.53 & 3.84 & 3.55 & 3.71 & 3.53 & 3.90 \\
\hline $\mathrm{T}_{1}\left({ }^{3} \pi \pi^{*}\right)$ & 2.71 & 2.51 & -0.20 & 2.46 & 2.49 & 2.39 & 2.50 & 2.72 \\
\hline $\mathrm{T}_{2}\left({ }^{3} \mathrm{n} \pi^{*}\right)$ & 3.23 & 3.80 & +0.57 & 3.74 & 3.48 & 3.63 & 3.46 & 3.83 \\
\hline $\mathrm{T}_{3}\left({ }^{3} \pi \pi_{2}\right)$ & 3.57 & 3.59 & +0.02 & 3.55 & 3.59 & 3.53 & 3.61 & 3.31 \\
\hline $\mathrm{T}_{4}\left({ }^{3} \pi \pi^{*}\right)$ & 3.60 & 3.54 & -0.05 & 3.58 & 3.77 & 3.76 & 3.81 & 3.85 \\
\hline $\mathrm{T}_{5}\left({ }^{3} \pi \pi_{4}\right)$ & 3.73 & 3.69 & -0.04 & 3.67 & 3.97 & 3.69 & 4.00 & 3.98 \\
\hline
\end{tabular}

Table 2: Adiabatic and 0-0 energies of the relevant states. Values are given in $\mathrm{eV}$.

\begin{tabular}{|l|r|r|r|r|r|}
\hline & \multicolumn{1}{|c|}{$\mathrm{S}_{1}\left({ }^{1} \pi \pi^{*}\right)$} & \multicolumn{1}{c|}{$\mathrm{S}_{2}\left({ }^{1} \mathrm{n} \pi^{*}\right)$} & \multicolumn{1}{c|}{$\mathrm{T}_{1}\left({ }^{3} \pi \pi^{*}\right)$} & $\mathrm{T}_{2}\left({ }^{3} \mathrm{n} \pi^{*}\right)$ & \multicolumn{1}{c|}{$\mathrm{T}_{3}\left({ }^{3} \pi \pi^{*}\right)$} \\
\hline adiabatic energy & 3.06 & 3.55 & 2.39 & 3.46 & 3.31 \\
\hline 0-0 energy & 2.93 & 3.46 & 2.28 & 3.38 & 3.17 \\
\hline
\end{tabular}




\section{References}

1. Mundt, R.; Villnow, T.; Ziegenbein, C. T.; Gilch, P.; Marian, C.; Rai-Constapel, V., Thioxanthone in Apolar Solvents: Ultrafast Internal Conversion Precedes Fast Intersystem Crossing. Phys. Chem. Chem. Phys. 2016, 6637-6647.

2. Onidas, D.; Markovitsi, D.; Marguet, S.; Sharonov, A.; Gustavsson, T., Fluorescence Properties of DNA Nucleosides and Nucleotides: A Refined Steady-State and Femtosecond Investigation. J. Phys. Chem. B 2002, 106, 11367-11374.

3. Kubista, M.; Sjöback, R.; Eriksson, S.; Albinsson, B., Experimental Correction for the InnerFilter Effect in Fluorescence Spectra. Analyst 1994, 119, 417-419.

4. Fröbel, S.; Buschhaus, L.; Villnow, T.; Weingart, O.; Gilch, P., The Photoformation of a Phthalide: A Ketene Intermediate Traced by FSRS. Phys. Chem. Chem. Phys. 2015, 17, 376-386.

5. Mooney, J.; Kambhampati, P., Get the Basics Right: Jacobian Conversion of Wavelength and Energy Scales for Quantitative Analysis of Emission Spectra. J. Phys. Chem. Lett. 2013, 4, 3316-3318. 6. Parson, W. W., Modern Optical Spectroscopy, with Exercises and Examples from Biophysics and Biochemistry, Student Edition; Springer: Dordrecht, Heidelberg, London, New York, 2009.

7. Strickler, S.; Berg, R., Relationship between Absorption Intensity and Fluoresence Lifetime of Molecules. J. Chem. Phys. 1962, 37, 814-822.

8. Reiffers, A.; Torres Ziegenbein, C.; Engelhardt, A.; Kühnemuth, R.; Gilch, P.; Czekelius, C., Impact of Mono-Fluorination on the Photophysics of the Flavin Chromophore. Photochem. Photobiol. 2018, 94, 667-676.

9. Zhou, W.; Yang, Y.; Liu, Y.; Deng, G.-J., Copper-Catalyzed C-C Bond Cleavage and Intramolecular Cyclization: An Approach toward Acridones. Green Chem. 2013, 15, 76-80.

10. Andronico, L. A.; Quintavalla, A.; Lombardo, M.; Mirasoli, M.; Guardigli, M.; Trombini, C.;

Roda, A., Synthesis of 1,2-Dioxetanes as Thermochemiluminescent Labels for Ultrasensitive Bioassays: Rational Prediction of Olefin Photooxygenation Outcome by Using a Chemometric Approach. Chem. Eur. J. 2016, 22, 18156-18168.

11. Maegawa, Y.; Mizoshita, N.; Tani, T.; Inagaki, S., Transparent and Visible-Light Harvesting Acridone-Bridged Mesostructured Organosilica Film. J. Mater. Chem. 2010, 20, 4399-4403.

12. Villnow, T.; Ryseck, G.; Rai-Constapel, V.; Marian, C. M.; Gilch, P., Chimeric Behavior of Excited Thioxanthone in Protic Solvents: I. Experiments. J. Phys. Chem. A 2014, 118, 11696-11707. 13. Carmichael, I.; Helman, W. P.; Hug, G. L., Extinction Coefficients of Triplet--Triplet Absorption Spectra of Organic Molecules in Condensed Phases: A Least-Squares Analysis. J. Phys. Chem. Ref. Data 1987, 16, 239-260.

14. Turbomole V7.5 2020, a Development of University of Karlsruhe and Forschungszentrum Karlsruhe GmbH, Turbomole GmbH, since 2007; Available from https://www.turbomole.org. 19892007.

15. Neugebauer, J.; Reiher, M.; Kind, C.; Hess, B. A., Quantum Chemical Calculation of Vibrational Spectra of Large Molecules - Raman and IR Spectra for Buckminsterfullerene. J. Comput. Chem. 2002, 23, 895-910.

16. Etinski, M.; Tatchen, J.; Marian, C. M., Time-Dependent Approaches for the Calculation of Intersystem Crossing Rates. J. Chem. Phys. 2011, 134, 154105.

17. Grimme, S.; Waletzke, M., A Combination of Kohn-Sham Density Functional Theory and Multi-Reference Configuration Interaction Methods. J. Chem. Phys. 1999, 111, 5645-5655.

18. Marian, C. M.; Heil, A.; Kleinschmidt, M., The DFT/MRCI Method. WIREs Comput. Mol. Sci. 2019, 9, e1394.

19. Kleinschmidt, M.; Tatchen, J.; Marian, C. M., Spin-Orbit Coupling of DFT/MRCI 
Wavefunctions: Method, Test Calculations, and Application to Thiophene. J. Comput. Chem. 2002, 23, 824-833.

20. Heß, B. A.; Marian, C. M.; Wahlgren, U.; Gropen, O., A Mean-Field Spin-Orbit Method Applicable to Correlated Wavefunctions. Chem. Phys. Lett. 1996, 251, 365-371.

21. Bracker, M; Marian, C. M.; Keinschmidt, M., Internal conversion of singlet and triplet states employing numerical DFT/MRCI derivative couplings: Implementation, tests and application to xanthone. Submitted to J. Chem. Phys. 